2) When the arrest time exceeded over 10 minutes the increased vascular resistance was striking. After reopening of the circulation the volume of the inflow (a) reduced further, or (b) restored slowly, or (c) increased considerably. In the cases of (a) and (c) the electrical activity seldom reappeared or reappeared incompletely after long time lapse. Only in the case of (b) the EEG reappeared and grew to the prior pattern. The gradual decrease of flow as in (a) could be inhibited and vascular resistance could be reduced to the prior value by infusion of a considerable quantity of the artificial solution. The EEG's reappeared in this circumstance. In the case of such as (a) the increase of the flow could be produced by elevating the perfusion pressure, but the EEG did not reappear. In the latter case, the blood pressure of the circle of Willis was rather lower to the perfusion pressure and oxygen contents of the internal cerebral vein was very high.

The facts stated above indicate that hindrance of blood flow to the nervous elements resulted from mechanical obstruction of the supply vessels and opening of shunt routes could be attributed to an origin of loss of brain function following circulatory arrest to the brain.

\title{
25. Experimental Transplantation of the Head
}

\author{
—Two Headed Dog-
}

Keiji Sano, Hideo Terao, Isao Hayakawa, Shuji Kamano and Isamu Saito

Dept. of Neurosurgery, Univ. of Tokyo

(See the text)

\section{Complication, Efficiency and Applycation of Tracheotomy in Neurosurgical Diseases}

\author{
Hachiro Uchiyama, Tetsuo ShIRao, Hitoshi Ichiki, Kazuo Uchiyama, \\ Yoshiaki OKuma and Akinori Nakamura \\ Ist Department of Surgery, Faculty of Medicine, Kagoshima University
}

For the past few years, we have experienced a 10 percent decrease in moritality following major neurosurgical procedures. It is our opinion that 
this decrease is related to an increase in the number tracheotomy performed. This contents in 43 patients with tracheotomy were analyzed. We feel that tracheotomies have a particularly important role in the post-operative management of patients subjected to hypothermia. Although we stress the importance of tracheotomy, drying and infection of the tranchea were troublesome complications in many patients. As a result, we investigated the complications associated with tracheotomies.

A few rare cases of these complications will be reported. A six monthold baby with hydrocephalus died of suffocation when the tip of a No. 4 tracheotomy canule impinged upon the carina of the trachea with a resulted blockage of the trachea from secretions. Three patients died of suffocation from ulcus formed in the anterior tracheal wall with resulted hemorrhage.

\title{
27. Postoperative Management by Rewarming from Hypothermia in Brain Operation
}

\author{
Kenzo Matsuoka, Eizo OKamoto, Toru Uozumi, Ryoichi Nozaki, \\ Syunnosuke Minami, Muneo Kazimura, Mitsumasa Kano, \\ Saburo SAKaKI, Masanori Nanba, Takashi Katsuda \\ and Sataro JitsukAWA \\ First Depertment of Surgery, Osaka University Medical School
}

The superiority of operation under hypothermia is well recognized in neurosurgical field. It is however, still open to controversy that what is the most optimal method of rewarming after operation under hypothermia from a view point of postoperative management.

Since 1955 , we have employed slow rewarming, that maintain hypohermia between $30^{\circ} \mathrm{C}$ and $34^{\circ} \mathrm{C}$ after operation and rewarm the patient ;lowly in 12 to 72 hours postoperatively. We have been able to control the sostoperative cumbrous complication such as brain edema, rebleeding and nyperthermia, and favourable results were obtained.

We applied this method in 24 cases, that included 62 cases of brain tumors, + cases of cerebral vascular diseases, 2 cases of brain abscess, 4 cases of ievere head injuries and 2 cases of other cerebral diseases. Six cases died n one month after operations. Operative mortality was $8.1 \%$. 